\title{
APPLICATION POTENTIAL OF NANOCRYSTALLINE RIBBONS STILL PENDING
}

\author{
Pavol Butvin * Beata Butvinová ${ }^{*}$ _ Peter Švec ${ }^{*}$ _ Jozef Sitek ${ }^{* *}$
}

\begin{abstract}
Nanocrystalline soft-magnetic ribbons promised a wide-spread practical use when introduced at the beginning of nineties. After 20 years of extensive research there are still unclear material problems which are thought to be the principal reason why these materials show but marginal use. Poorly controllable magnetic anisotropy due to spontaneous intrinsic macroscopic stress that comes from an inevitable heterogeneity of the ribbon materials is pointed to in this work. Certain stress-based mechanisms are shown to induce the unintended anisotropy in the already familiar Finemets as well as in the newer Hitperms. Hysteresis loops, domain structure and power loss is used to reveal the anisotropy consequences and particular connected but still unanswered questions are pinpointed.
\end{abstract}

K e y w or ds: soft-magnetic materials, nanocrystalline ribbons, magnetic anisotropy, hysteresis loop, power loss

\section{INTRODUCTION}

Soft-magnetic ribbons produced by rapid quenching from the melt are already used in the industrial practice. Magnetic properties of specific compositions greatly profit from the thermal treatment that leads to partial crystallization where nano-scale crystalline grains are embedded in the amorphous rest of the alloy. As for these nanocrystalline materials, the product range of major manufacturers shrunk in the last years - the only composition used in the current larger-scale production is Fe-Nb-Cu-B-Si with $15.5 \div 16$ at. \% Si. Apart from commercial reasons there are still certain physical properties, where the lack of detailed knowledge limits the use of nanocrystalline ribbon materials. A class of the abovementioned properties are those, which lead to the generation of intrinsic macroscopic stress. Such stress is capable of contributing significant magnetic anisotropy. The magnetoelastic contribution due to the stress is well documented but other mechanisms are possible too. As for the sources of the stress, the so-called macroscopic heterogeneity $(\mathrm{MH})[1]$ is considered in this work. $\mathrm{MH}$ is the term [2] we propose to encompass certain effects connected to: "native oxide layer" [3], "different SRO at the surfaces" [4] and "surface crystallization" [5]. The quoted terms have something common - all point to a largescale heterogeneity across the ribbon thickness, ie to various differences between the surfaces (surface-adjacent layers) and the deeper layers (interior). Another essential common point: No thermal treatment makes the obviously as-cast-based heterogeneity disappear - it merely transforms [6] to give different outcome in different annealing ambience. The term MH should promote as well the distinction from short-range heterogeneity (nanocrystals, interfaces, clusters, rare solitary defects ... ). As all materials with a large surface-to-volume ratio, the rib- bons show more or less MH. The possibility to tailor the soft-magnetic properties is often limited by MH. The ribbons often show significant dimension change during thermal treatment. Another stress can build up if a more massive component (eg tape-wound core) is submitted to inhomogeneous heating. Nevertheless, some materials $(e g$ the more recent Fe-Co-Nb-B Hitperms) respond to modest stressing above $\sim 400{ }^{\circ} \mathrm{C}$ by viscous flow and show rather ambiguous outcome as to the expected anisotropy contribution. Thus there are still particular properties of nanocrystalline ribbons, which are not clear enough to enable more intensive practical use. In this paper, simple summary for the role of MH in Si-poor Finemets is presented and certain problems of $\mathrm{MH}$ influence is pointed to for the other nanocrystalline ribbon materials.

\section{EXPERIMENTAL}

The ribbons of Finemets (Fe-Nb-Cu-B-Si; see Table 1 for detailed composition) and Hitperm $\left(\mathrm{Fe}_{61} \mathrm{Co}_{20} \mathrm{Nb}_{7} \mathrm{~B}_{12}\right)$ were prepared by planar-flow casting in air. The strip samples were cut from $17 \div 25 \mu \mathrm{m}$ thick and $10 \mathrm{~mm}$ wide as-cast ribbons to give a length of $100 \mathrm{~mm}$. Toroidal cores showed $30 \mathrm{~mm}$ inner diameter and an average mass of $10 \mathrm{~g}$. Only $19 \div 20 \mu \mathrm{m}$ thick ribbons were wound to toroids for the loss measurement. The target magnetic properties together with the nanocrystalline state were obtained by annealing at $540^{\circ} \mathrm{C}$ for 1 or 2 hours (except indicated otherwise) in different ambiences. Annealing was performed in vacuum, in pure Ar (with the furnace evacuated before Ar was let in) and in "technical" Ar (without preceding evacuation) or in air. The hysteresis loops were recorded at room temperature by a digitizing hysteresisgraph. J-H loops for the strips were measured in Helmholtz coils at $21 \mathrm{~Hz}$ sine-H excitation, whilst B-H

\footnotetext{
* Institute of Physics SAS, Bratislava, Slovakia, pavol.butvin@savba.sk ${ }^{* *}$ Department of Nuclear Physics \& Technology, FEI, Slovak Technical University, Bratislava, Slovakia
} 


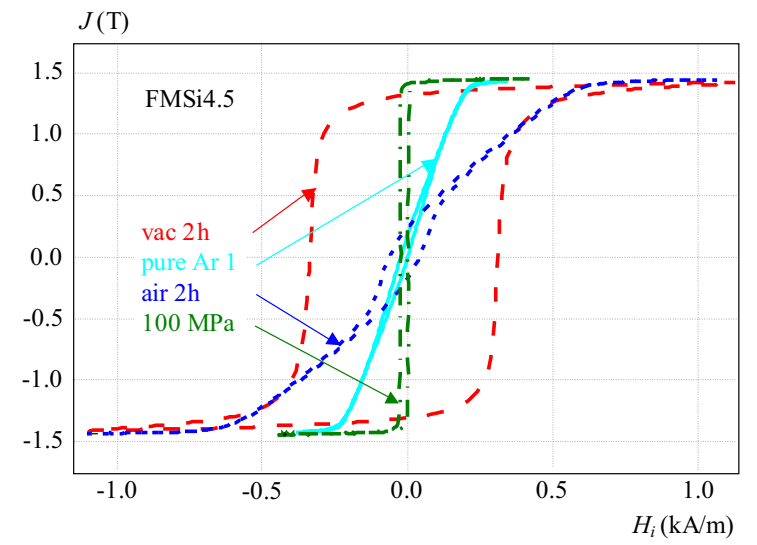

Fig. 1. Hysteresis loops of strip samples annealed at $540^{\circ} \mathrm{C}-$ technical Ar atmosphere was used at $100 \mathrm{MPa}$ longitudinal-stress annealing with $0.5 \mathrm{~h}$ isotherm duration

Table 1. Summary of Finemet composition, magnetostriction, magnetization work and power loss after Ar-annealing

\begin{tabular}{lcccc}
\hline Alloy & $\lambda_{s}$ & $W_{m}$ & $\mathrm{pFe}$ & $\mathrm{pFe}$ \\
composition & $10^{-6}$ & $\mathrm{~J} / \mathrm{m}^{3}$ & $2 \mathrm{kHz}$ & $100 \mathrm{kHz}$ \\
\hline Fe73.5 Nb3 Cu1 B6.5 Si16 & & & \\
FMSi16 & -2.9 & 4.8 & 0.28 & 91 \\
Fe73.5 Nb3 Cu1 B7 Si15.5 & & & \\
FMSi15.5 & 2.6 & 4.0 & 0.22 & 73 \\
FeNbCuBSi & Vitroperm 800F B & & \\
VP800F & - & 17.7 & 0.17 & 93 \\
Fe73.5 Nb3 Cu1 B9.5 Si13 & & & \\
FMSi13 & 3.3 & 2.5 & 0.36 & 102 \\
Fe75 Nb3 Cu1 B12 Si9 & & & \\
FMSi9 & 8.0 & 36 & 0.46 & 147 \\
Fe78 Nb3 Cu1 B13.5 Si4.5 & & & \\
FMSi4.5 & 10.2 & 175 & 0.90 & 212 \\
\hline \multicolumn{5}{c}{} \\
\hline
\end{tabular}

lops were recorded for the toroids (sine-B mode was attained at a medium induction from $210 \mathrm{~Hz}$ to $100 \mathrm{kHz}$ ). Core loss was determined at $0.3 \mathrm{~T}$ induction from the B$\mathrm{H}$ loop area on toroids only. The magnetic domains were observed on strips by digitally enhanced Kerr microscopy [7]. Mössbauer spectroscopy used standard technique [8] to look for response of excited ${ }^{57} \mathrm{Fe}$ nuclei within the alloy. It operated in transmission geometry (TMS) to record the response from the volume and in reflective geometry (CEMS) where conversion electrons bring the response from surface-adjacent layers only.

\section{RESULTS AND DISCUSION}

The majority of unresolved problems of nanocrystalline magnetic ribbons point to $\mathrm{MH}$ as the most important factor. Whereas the role of $\mathrm{MH}$ in Si-poor Finemets seems to be relatively clear, Si-rich Finemets and newer materials (eg Hitperms) still present several unclear questions. Relatively strong creep-induced magnetic anisotropy and the MH-induced macroscopic stress interacting with ambiguous magnetostriction represent the main uncertainty for Si-rich Finemets, the viscous flow provides unresolved consequences for the anisotropy of Hitperms.

\subsection{Finemets}

\subsubsection{Magnetic anisotropy and macroscopic} heterogeneity of Si-poor Finemets

Si-poor Finemets (and many other similar materials) are prone to surface crystallization to the detriment of interior crystallization as shown by TMS\&CEMS and ferromagnetic resonance / antiresonance [1]. This "differential" surface change at annealing often produces an inplane stress $\sigma$, which couples to magnetostriction $\lambda$ and generates hard-ribbon-axis (HRA) magnetic anisotropy. Its coefficient $K_{\mathrm{HRA}}$ can be expressed as

$$
K_{\mathrm{HRA}}=3 / 2(\lambda \times \sigma) \sim W_{m}
$$

and can attain hundreds of $\mathrm{J} / \mathrm{m}^{3}$. The coefficient is proportional to the so-called magnetization work $W_{m}[9]$ which can be calculated from the loop tilt (quoted in Table 1). It can be used to estimate the stress $\sigma$ if a known stress acts on a possibly homogeneous sample to match $W_{m}$ of a comparable heterogeneous sample [1] $-\sigma$ can attain up to $70 \mathrm{MPa}$ in the ribbon interior if it represents some $80 \%$ of the ribbon thickness. Result of air (similar to "technical" Ar) annealing compared to vacuum annealing is seen for Si-poor Finemet in Fig. 1.

Ar or air annealing produces the MH-induced HRA anisotropy (tilted loops) and short-duration vacuum annealing leads to a slim upright loop (just like the one shown for stress annealing). Since the difference in (average) magnetostriction after vacuum and Ar annealing is insignificant, vacuum annealing appears to produce feebler $\mathrm{MH}$ or at least weaker stress. Another effect demonstrates after comparable but prolonged vacuum annealing ("thick" loop). Magnetically hard borides have been identified in the vacuum annealed sample. Thus it appears as if $\mathrm{MH}$ is capable of blocking the crystallization of the borides in certain range of annealing temperatures and durations.

Domain structure of FMSi4.5 shown in Fig. 2 a) to c) reflects well the difference of the diversely annealed samples. The vacuum-annealed sample with borides shows typical magnetically-hard domain structure - it was confirmed by observing the response of the structure to bias field. Fig. 2b) shows so-called baked-in [7] domains and refers to the most slant loop of Fig. 1. Whereas $130 \div 150 \mathrm{~A} / \mathrm{m}$ of longitudinal bias field is enough to decrease the contrast substantially (rotational processes are most probable), the tiny diagonal domains could be distinguished unaltered to a manifold stronger field. The diagonal domains closely resemble closure domains which emerge under stress and are thought to be supported by atomic structure stabilized under the stress already at 


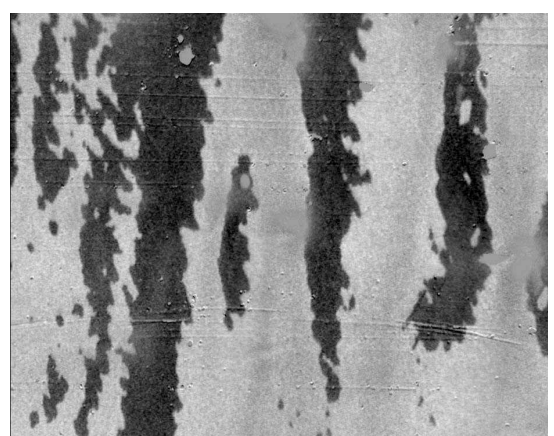

(a)

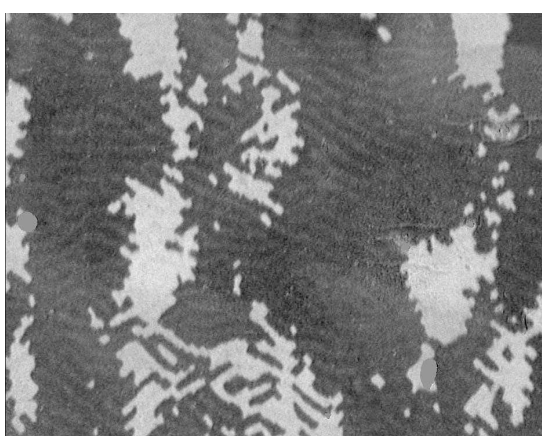

(b)

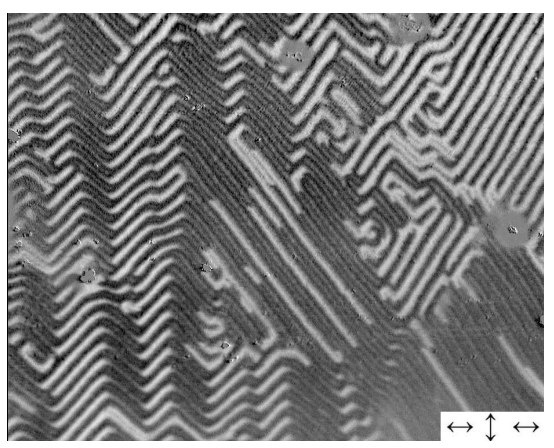

(c)

Fig. 2. Domain structure of FMSi4.5 diversely annealed at $540^{\circ} \mathrm{C}$. Left to right: a) $2 \mathrm{~h}$ in vacuum; b) $2 \mathrm{~h}$ in air; c) $1 \mathrm{~h}$ in pure Ar. Displayed field actual horizontal dimension is $0.5 \mathrm{~mm}$, the arrows indicate for all images: ribbon axis; maximum magneto-optical sensitivity; dc bias field (zero in c))

Table 2. Mössbauer spectroscopy parameters of Si-rich Finemets

\begin{tabular}{lcccc}
\hline $\begin{array}{l}\text { Alloy label; } \\
\text { ann. }\end{array}$ & $\begin{array}{c}\text { vol./ } \\
\text { surf. }\end{array}$ & $\begin{array}{c}\langle\text { Bhf }\rangle \\
(\mathrm{T})\end{array}$ & $\begin{array}{c}\text { Acr } \\
(\%)\end{array}$ & DO3 \\
\hline FMSi16; vac & $\mathrm{V}$ & 17.2 & 63.8 & good \\
& $\mathrm{S}$ & 20.3 & 44.9 & good \\
\hline FMSi16; Ar & $\mathrm{V}$ & 20.5 & 47.0 & good \\
& $\mathrm{S}$ & 19.1 & 46.6 & poor \\
\hline FMSi15.5; vac & $\mathrm{V}$ & 18.0 & 50.1 & good \\
& $\mathrm{S}$ & 18.8 & 43.1 & poor \\
\hline FMSi15.5; Ar & $\mathrm{V}$ & 17.4 & 58.1 & good \\
& $\mathrm{S}$ & 19.8 & 44.1 & none \\
\hline FMSi13; vac & $\mathrm{V}$ & 19.7 & 38.6 & good \\
& $\mathrm{S}$ & 21.6 & 36.0 & none \\
\hline
\end{tabular}

high enough temperature. No magnetically harder material (borides) has been identified in such samples. Resilient but responding surface-domain structure is seen in Fig. 2c) for the sample annealed in pure Ar. It is again a sort of closure domains since they are magnetized "headon" [7]. Although narrow surface domains are discernible at a larger bias field than is necessary to alter the background saturation visibly, the domains start to change simultaneously with the background-saturation change.

Apart from strong-anisotropy borides and magnetoelastic (ME) anisotropy induced by final (room-temperature) state of $\mathrm{MH}$, there is still other stress-based mechanism capable of creating magnetic anisotropy. A macroscopic stress with defined geometry acting at high enough temperature and kept acting until the sample cools down can establish a strong anisotropy of its own - the socalled creep-induced (CI) anisotropy. This anisotropy is of easy-ribbon-axis type (see the upright loop in Fig. 1) up to $\sim 8$ at. $\%$ of $\mathrm{Si}$ and reverts to transverse anisotropy (not HRA!) at a higher than $10 \% \mathrm{Si}$ percentage when the stress is tensile along the ribbon axis [10]. However if the surfaces exert the $\mathrm{MH}$-typical compressive in-plane stress, the resulting CI anisotropy of the ribbon interior would be just of the HRA type and thus it would add to the ME anisotropy of the positively magnetostrictive Si-poor
Finemets. The equal sign of ME and CI anisotropy makes it difficult to discern these components. We only cannot exclude the presence of the MH-induced CI anisotropy at the lower end of Si percentage, where CI is strong enough. All the Finemets decrease its magnetostriction with advanced annealing (until the second-stage crystallization starts) [10]. This trend is followed by the anisotropy of MH-influenced and CI-free FMSi9 but not by that of FMSi4.5 [11] and this fact strongly points to the presence of same-sign CI anisotropy in FMSi4.5. The assortment of variable loops (Fig. 1) shows tailoring capability and points to some application potential of Si-poor Finemets - eg using a reasonably linear loop for sensors or chokes. Unfortunately, the relatively high magnetostriction (Table 1) and large coercivity (the lowest value is $5 \mathrm{~A} / \mathrm{m}$ for FMSi9 toroid) is hardly balanced by the approximate loop linearity and somewhat higher saturation of Finemets with less than 10 at.

\subsubsection{Magnetic anisotropy and macroscopic heterogeneity of Si-rich Finemets}

We name here the Finemet compositions containing 12 to 16 at.\% Si the Si-rich Finemets. Great majority of practically applied nanocrystalline soft-magnetic materials belongs here. The low magnetostriction $\left(\left|\lambda_{s}\right|<\right.$ $\left.3 \times 10^{-6}\right)$ came out as the crucial parameter from the application viewpoint. Nevertheless, still there are problems with reproducibility and the ensuing magnetic parameter spread. This well could mean that some material properties remain out of control or even out of proper attention. $\mathrm{MH}$ is such a property. One of the methods of choice to reveal the presence of MH is the TMS/CEMS combination (see Table 2).

TMS points to a well developed DO3 superstructure of crystalline grains within the major part of responding ribbon volume. The optimal stoichiometry for the DO3 were $\mathrm{Fe}_{3} \mathrm{Si}$, thus 25 at.\% of $\mathrm{Si}$ in the grains. The evaluation of CEMS spectra seldom gives good fit when looking for the characteristic DO3 spectral components. In Table 2, the column label "DO3" means in fact, how good 


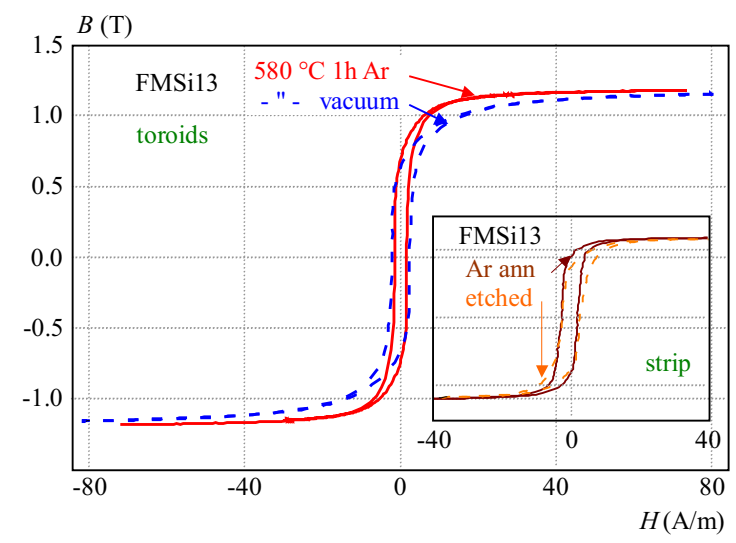

Fig. 3. Hysteresis loops of FMSi13 - the inset displays loops prior to and after surface removal by etching, "Ar" means technicalpurity Ar here

is the fit of measured spectrum when a superposition of DO3 components is assumed. Considering eg the XPS results [6] or corrosion studies $[8,12]$, silicon is seen to engage in $\mathrm{SiO}_{x}$ oxides and its availability for the DO3forming $\mathrm{Fe}_{3} \mathrm{Si}$ is thus limited at ribbon surfaces. In the same spirit of oxidation chance for silicon, the surfaces of Ar- or air-annealed samples are more DO3-poor than the vacuum-annealed ones. Another modest surface-tointerior difference is observed in hyperfine field $\langle\mathrm{Bhf}\rangle$ too where different values reflect different neighborhood of ${ }^{57} \mathrm{Fe}$ nuclei remaining in amorphous rest of the alloy. This field is mostly stronger at the surfaces. However contrary to Si-poor Finemets, there is no prevalence of surface crystallization in Si-rich Finemets as seen if the fraction of ${ }^{57} \mathrm{Fe}$ nuclei in crystalline grains (represented by Acr) is compared between volume and surfaces. All these observations point to $\mathrm{MH}$ being present in Si-rich Finemets too.

Though, a pronounced magnetoelastic effect (relation (1)) cannot be expected due to the low magnetostriction. Moreover, Si-rich Finemets containing 15 to 16 at.\% Si show low, but variable-sign magnetostriction (see Table 1) - it often depends on the annealing temperature and ambience used at thermal treatment. Thus the chance to identify the MH-typical in-plane stress by observing the ensuing ME contribution to anisotropy is limited. Nevertheless, the in-plane stress coming from MH could be significant and it can induce the above-mentioned CI anisotropy. Let us assume, the surfaces press on the interior (squeezing surfaces) already during the start of cooling down at the end of annealing. Then the resulting CI anisotropy would be of easy-ribbon-plane type and the strip sample geometry would further promote an easy-ribbon-axis type. If the same-type stress holds or re-establishes at the final temperature (room temperature), the ME anisotropy of a positively magnetostrictive material would be of just the opposite HRA type. This $\mathrm{ME} / \mathrm{CI}$ sign mismatch is limited to a narrow range of Si percentage (12-14\%) [10] in Finemets only. The mismatch provides the chance for the anisotropy contributions to compensate each other and if so, such materials can show magnetic anisotropy insensitive to $\mathrm{MH}$ - surely a welcome property from the application viewpoint. The simple idea to disclose the actual situation is to remove the surfaces. Figure 3 shows the result in the inset.

The comparison of Si-rich and Si-poor materials, not limited to just Finemets, shows the former to tilt the loop and the latter to erect the loop after the surface removal $[13,14]$. If the opposite response of the Si-rich Finemets actually comes from the removal of not squeezing, but spreading surfaces, then it means that $\mathrm{MH}$ actually can create stress of either sign. Thus despite of opposite sign of ME and CI anisotropy contributions in Si-rich Finemets, the either sign of stress again puts the question - MH-induced CI anisotropy present or absent - into a haze because the active in-plane stress has to be identified prior to evaluating the resulting anisotropy. There is still no work where the presence and evolution of an unintended macroscopic stress during the cooling of just annealed sample was identified. Apart from intentional stress annealing, the rare occasion to observe the remnants of high-temperature stress action (intermittent one inclusive) is when the domain structure shows the above-mentioned baked-in domains (Fig. 2). So far, we have observed this domain type in Si-rich Finemets but occasionally. Thus even very low anisotropy of a heterogeneous material cannot be seen as an effective zero anisotropy but rather as more or less balanced heterogeneous anisotropy of diverse origin. The main part of Fig. 3 shows loops of vacuum- and Ar-annealed toroidal samples compared. Similarly to the Mo-substituted Finemets [13], this FMSi13 Nb-Finemet shows clear difference albeit somewhat higher annealing temperature is necessary to get good reproducibility. The sense of the difference points to slightly stronger HRA (or transverse) anisotropy in vacuum annealed samples. This represents another contrast to the Si-poor Finemets. As there is no hint to assume stronger low-temperature macroscopic stress in vacuum annealed Si-rich Finemets, stronger MH-caused spreading of interior by surfaces in Ar-annealed samples appears to be the more probable explanation. This would agree with the sense of etching experiment and with an expected action of the "silicon-oxide layer" $[8,12]$. Unfortunately, we know very little about a possible hightemperature stress and this is especially important just in Si-rich Finemets since CI anisotropy could be far stronger than ME anisotropy in these low-magnetostrictive materials. This is the background of suspecting MH to tamper with the reproducibility of anisotropy of the most used Finemet.

\subsubsection{Power loss}

The low power loss is one of the most advantageous and most wanted properties of nanocrystalline materials. Figure 4 shows the comparison of power loss for three differently thermally treated toroids wound of the widely used Si-rich Finemet $\mathrm{Fe}_{73.5} \mathrm{Nb}_{3} \mathrm{Cu}_{1} \mathrm{~B}_{7} \mathrm{Si}_{15.5}$. Whereas the transverse (relative to ribbon) anisotropy of the 

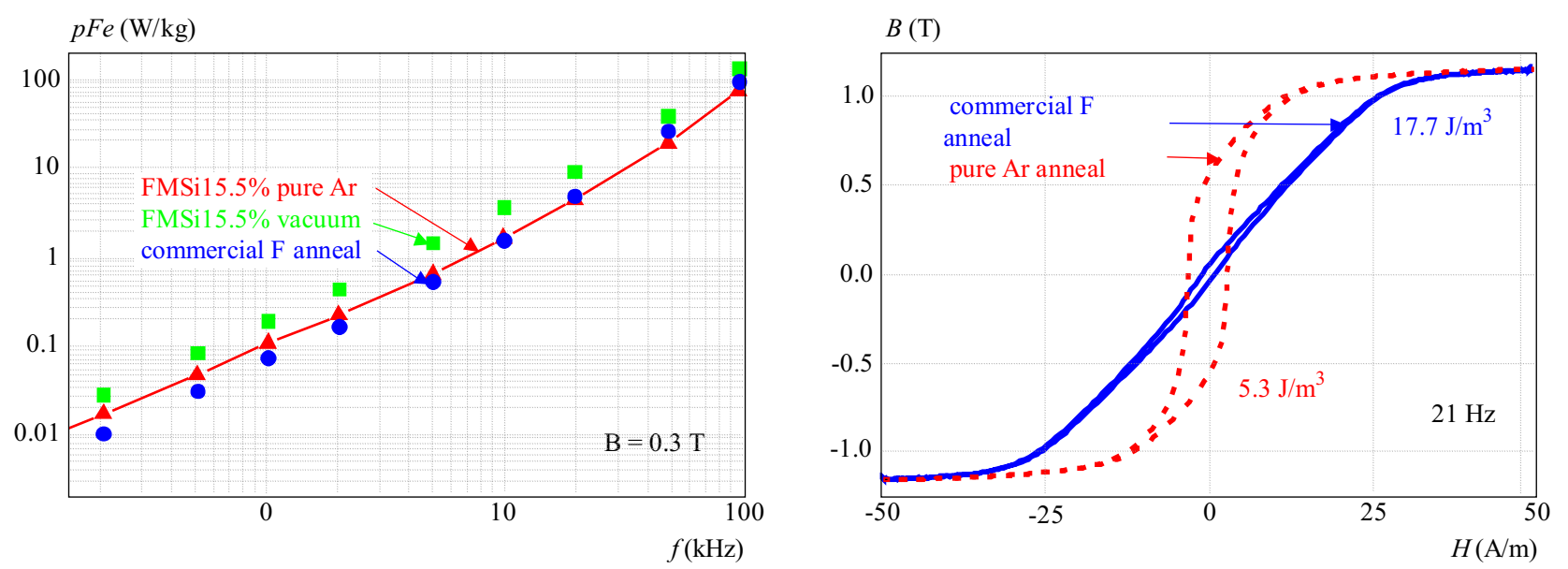

Fig. 4. Core loss frequency variation (upper graph) and hysteresis loops (lower graph) for FMSi15.5 measured at indicated conditions - the values in $\mathrm{J} / \mathrm{m}^{3}$ indicate magnetization work $W_{m}$, the connecting line is a mere help to resolve "the best"-loss points

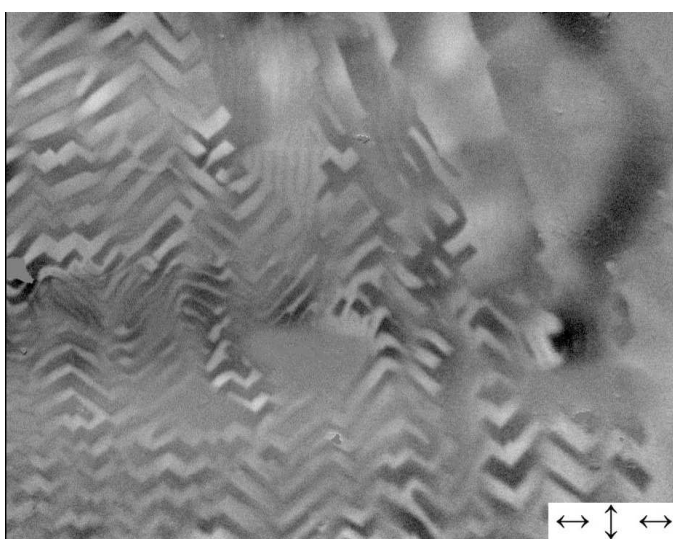

Fig. 5. Domain structure of FMSi15.5 strip annealed for $1 \mathrm{~h}$ at $540^{\circ} \mathrm{C}$ in pure $\mathrm{Ar}$ - the arrows mean the same as for Fig. 2

field-annealed core promotes magnetization rotation, the heterogeneous Ar-annealed material shows fine domain structure (Fig. 5.) and supports domain wall movement.

Thus the risk of magnetostrictive noise generation as well as of domain wall stabilization (baking-in) detrimental consequences is lowered. The comparison of vacuumand Ar-annealed cores shows slight but clear preferences for the Ar annealed one: apart from loss, the approach to saturation can be better tailored by varying the annealing duration. There are two more diversities observed. Unlike the pure-Ar annealed material (Table 1), the vacuumannealed one shows equally minor, but negative magnetostriction. The very similar $21 \mathrm{~Hz}$ loops (therefore just one displayed) differ from each other in the opposite sense if compared to FMSi13 (Fig. 3). All this points again to a fine but intricate influence of $\mathrm{MH}$ despite of low magnetostriction. Field-annealed "demonstration" commercial core shows lower loss at lower frequencies and higher induction. The "right amount" of MH in the no-field annealed core creates heterogeneous anisotropy, where a part of the core volume provides for even lower loss at

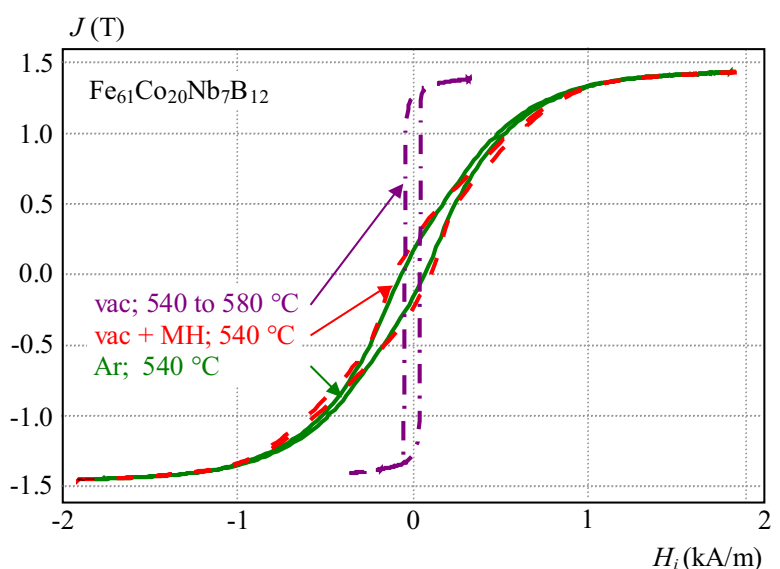

Fig. 6. Hysteresis loops of $1 \mathrm{~h}$ annealed Hitperm strips - some vacuum-annealed samples show the upright loop up to $580^{\circ} \mathrm{C}$, some tilt somewhat, none exceeds the tilt matching the loop of technicalAr-annealed sample

higher frequency up to $B=0.35 T$. There are two principal reasons to think of just a part of the core volume or, more precisely, of a part of ribbon cross section. Due to frequency-dependent flux penetration depth, a gradient of peak induction is created across the ribbon thickness at higher frequencies. Thus the central part of ribbon cross section starts to participate less on the average induction swing ( $0.3 \mathrm{~T}$ for our data) and the properties of the surface-adjacent parts come more into play. Unfortunately, the MH creates hysteretic surface domain structures [7]. Thus the surface layer created by $\mathrm{MH}$ should be optimized so as not to add to too much loss and still create the advantageous anisotropy deeper below the surfaces. (Indeed, we observe more flat loss versus frequency variations for $\mathrm{MH}$-affected Si-poor Finemets too, alas this effect is invalidated by unacceptably high overall loss see Table 1). Although the saturated loops in Fig. 4 inset differ grossly, the corresponding domain structure is not so much different at least in a part of the ribbon volume. Upper right corner of Fig. 5. shows transversal domains (similar as observed on F-annealed material [7]). Toward 
the ribbon edge (downward), closure domains typical of in-plane stressed material are seen. Thus Si-rich Finemets offer still more possibilities if the macroscopic heterogeneity is manipulated. To use the possibilities, more detailed knowledge about MH should be gained.

\subsection{Hitperm}

Since 2000 there is a new class of soft-magnetic nanocrystalline material containing an appreciable percentage of Co, called Hitperm [15]. It shows new application potential (higher-temperature stability) but less use yet. The macroscopic heterogeneity seems to play still greater role than in Finemets. Slant loops typical of $\mathrm{MH}$ are sometimes observed after vacuum annealing too. So far, it is not clear what is the principal reason of such not well reproducible behavior.

As shown in Fig. 6., hysteresis loops of the "exemplary" Hitperm sometimes differ from each other although the conditions of vacuum annealing is thoroughly controlled to be the same. The comparison to Ar-annealed samples (well reproducible loops) demonstrates that it is the $\mathrm{MH}$ that sometimes builds up during vacuum annealing too. Hitperms generally show a preference for surface crystallization [15]. However it is not proven whether this effect is the crucial reason because the material response is complicated by appreciable viscous flow observed under modest stress (a few $\mathrm{MPa}$ ) at temperatures not much below the crystallization [16]. Some flow still works within the amorphous remainder during and after the crystallization. The Si-free Hitperms investigated so far resemble the behavior of low-Si Finemets in that their MH-typical HRA anisotropy increases with prolonged or higher-temperature annealing. However the high magnetostriction of Hitperms [16] does not significantly decrease with advanced crystallization. This seems to point to steadily rising $\mathrm{MH}$-caused stress $\sigma$ (relation (1)) and, unlike the Finemets, the partaking of CI anisotropy is not necessary to explain the behavior. However the CI anisotropy exists in Hitperms too (the same type as eg in FMSi4.5) and its creation is reasonably expected to be supported by the viscous flow. If the flow is capable of relaxing the stress prior to the CI anisotropy is induced (or stabilized at decreasing temperature), then manipulating the flow capability by changing the composition could serve another tool to tailor the properties of Hitperms. For instance, added Si is observed to support the viscous flow [17]. Nevertheless toroids, as the device shape where most of the similar materials are practically exploited, show the response to annealing very similar to strips. There are specific stresses that can act during thermal treatment of more massive tape-wound cores. So far however, the viscous flow has not been seen to demonstrate correspondingly. To clear the question whether $\mathrm{MH}$ and/or the viscous flow actually can induce persisting CI anisotropy is again important and the problem is worth of further research.

\section{CONCLUSION}

Certain physical properties of nanocrystalline ribbons still pose problems, which hamper a more extended exploitation of these materials in industrial practice. The issues still worth of more research are:

- The sources of macroscopic heterogeneity and methods of its control.

- Material response to macroscopic stresses.

- Material response to surface removal by diverse methods.

Even if the materials contain extensively studied nanostructures, a deeper knowledge of macroscopic properties of the material is equally important and should be further paid attention.

\section{Acknowledgments}

This work was supported in part by the national grant agency VEGA under Grants no. 2/0156/08, 2/0157/08 and 1/0606/09. Support of the Agency of the Min. Edu. of the Slovak Republic for the Structural Funds of the EU (CEKOMAT I, ITMS 26240120006) as well as by the Centrum of Excellence "Nanosmart" is gratefully acknowledged. Domain structures were observed and evaluated by the courtesy and under the auspices of Dr Rudolf Schäfer in his laboratory in IFW Dresden, Germany.

\section{REFERENCES}

[1] Butvin, P.-BUtvinovÁ, B.-FRAit, Z.-SITEK, J.ŠVEC, P.: Magnetic Response of FeNbCuBSi RQ Ribbons to Bi-Axial Strain, Journal of Magnetism and Magnetic Materials 215-216 (2000), 293-296.

[2] BUtVinovÁ, B.-BUTVIN, P.-SCHÄFER, R. : Influence of Heterogeneity on Magnetic Response of Nanocrystalline Ribbons, Sensors and Actuators A 106 (2003), 52-55.

[3] JAIN, R.-SAXENA, N. S.-RAO, K. V. R.-AVASTHI, D. K.-ASOKAN, K.-SHARMA, S. K. : Characterization of Native Oxide Layers on Amorphous $\mathrm{Fe}_{73.5} \mathrm{Si}_{15.5} \mathrm{~B}_{7} \mathrm{Cu}_{1} \mathrm{Nb}_{3}$ before and after High-Energy Heavy Iron Irradiation by X-Ray Photoelectron Spectroscopy (XPS), Material Sciences and Engineering. A 297 (2001), 105-110.

[4] MIGLIERINI, M.-SEBERÍNI, M.-TÓTH, I.-VITÁZEK, K.: Magnetic Microstructure on Surface and in Bulk of NANOPERM-Type Nanocrystalline Alloys: Mössbauer Effect Studies, Journal of Magnetism and Magnetic Materials 265 (2003), 243-247.

[5] HERZER, G.-HILZINGER, H. R. : Surface Crystallization and Magnetic Properties of Iron-Based Metallic Glasses, Physica Scripta 39 (1989), 639-641.

[6] CHenAKIN, S. P.-GAlstyan, G. G.-TOLSTOGOUZOV, A. B.-KRUSE, N.: XPS and ToF-SIMS Characterization of aFinemet Surface: Effect of Heating, Surface and Interface Analysis 41 No. 3 (2009), 231-237.

[7] HUBERT, A.-SCHÄFER, R. : Magnetic Domains, SpringerVerlag, Berlin, 1998, pages 35,445.

[8] SITEK, J.-SEDLAČKOVÁ, K.-SEBERÍNI, M. : Atmospheric Corrosion of Different Fe-Based Alloys in Nanocrystalline State, Czechoslovak Journal of Physics 55, No. 7 (2005), $883-891$. 
[9] CHIKAZUMI, S. : Physics of Magnetism, J. Wiley \& Sons, New York, 1964, page 16

[10] HERZER, G. : Nanocrystalline Soft Magnetic Alloys, Handbook of Magnetic Materials, Vol. 10 (K. H. J. Buchow, ed.), Elsevier Science B. V., 1997.

[11] BUtVin, P.—BUTVinovÁ, B.-DUHAJ, P.—VLASÁK, G. -ŠVEC, P.-JANIČKOVIČ, D.: Distinctive Annealing Behaviour of Si-Poor FeNbCuBSi Alloys, Materials Science and Engineering A 226-228 (1997), 659-662.

[12] SOUZA, C. A. C.-DE OLIVEIRA, M. F.-MAY, J. E.-BOTTA, F. W. J.-MARIANO, N. A.-KURI, S. E.-KIMINAMI, C. S.: Corrosion Resistance of Amorphous and Nanocrystalline Fe-M-B $(\mathrm{M}=\mathrm{Zr}, \mathrm{Nb})$ alloys, Journal of Non-Crystalline Solids 273 (2000), 282-288.

[13] BUtVin, P.-BUTVINovÁ, B.-SILVEYRA, J. M.CHROMČÍKOVÁ, M.-JANIČKOVIČ, D.-SITEK, J.ŠVEC, P.-VLASÁK, G.: Effect of Substitution of Mo for $\mathrm{Nb}$ on Less-Common Properties of Finemet Alloys, Journal of Magnetism and Magnetic Materails 322 (2010), 3035-3040.

[14] BUtvin, P.—BUTVinovÁ, B.—ŠVEC, P.—VLASÁK, G.JANIČKOVIČ, D.: Magnetic properties of Fe-Co-Mo-Cu-B nanocrystalline ribbons with stressing surfaces, Journal of Alloys and Compounds (2010), accepted, in press.

[15] WILlARD, M. A.-HUANG, M-Q.-LAUGHLIN, D. E.-MC HENRY, M. E.-CROSS, J. O.-HARRIS, V. G.-FRANCHETTI, C.: Amorphous/Nanocrystalline Materials - Magnetic Properties of HITPERM (Fe, Co)88Zr7B4Cu1 Magnets, Journal of Applied Physics 85 (1999), 4421-4423.

[16] BUtvin, P.-BUtVinovÁ, B.-SITEK, J.-DEGMOVÁ, J.-VLASÁK, G.- ک̌VEC, P.-JANIČKOVIČ, D. : Magnetic properties and macroscopic heterogeneity of FeCoNbB Hitperms, Journal of Magnetism and Magnetic Materials 320 (2008), 1133-1140.

[17] BUtVinová, B.-BUTVIN, P.-VLASÁK, G.-ŠSEC, P.JANIČKOVIČ, D.: Secondary magnetic anisotropy in Hitperms, Reviews on Advanced Materials Science 18 (2008), $509-512$.

Received 30 June 2010

Pavol Butvin (RNDr, CSc), born in Bratislava 1948, graduated from the Faculty of Natural Sciences, J.A. Comenius University Bratislava in 1971 and joined the Institute of
Physics, Slovak Academy of Sciences Bratislava. He acquired his RNDr. and CSc grades both in 1980 . He was member of the group awarded by State Prize in 1988. His interests are in magnetism of metals as well as in applied research of novel magnetic materials.

Beata Butvinová (RNDr, CSc), maiden name de Ronzyová, born in Nitra 1955, graduated from the Faculty of Natural Sciences, J.A. Comenius University Bratislava, from the branch of Experimental Physics in 1980. Then, she joined the Institute of Physics, Slovak Academy of Sciences Bratislava. She received RNDr and CSc grades in 1981 and 1987, respectively. Her current interests are in research of soft-magnetic properties of materials prepared by rapid quenching of the melt.

Peter Švec (Ing, DrSc), born in Bratislava, Czechoslovakia, in 1955, graduated from the Faculty of Electrical Engineering, Slovak University of Technology, Bratislava, from Solid State Physics branch, in 1979, and received the PhD degree in Physics in 1986. At present he is with the Institute of Physics, Slovak Academy of Sciences. His research field is the physic of metals, recently namely phase transformations in metal-metalloid glassy systems.

Jozef Sitek (Prof, Ing, DrSc), born in Záhorská Ves, Slovakia, in 1944, graduated from the Faculty of Electrical Engineering, Slovak University of Technology, in 1966. The PhD degree received in Applied Physics, in 1974 and DrSc degree in Physics of Condensed Matter, in 1994. At present he is Professor for Condensed Matter Physics at the Department of Nuclear Physics and echnology, Faculty of Electrical Engineering and Information Technology. The main field of his research and teaching activities are experimental nuclear physics, spectroscopy method, mainly application of Mössbauer spectroscopy.

This work was presented at Nanoved \& Nanotech \& Techtransfer 2010, International Conference on Nanoscience, Nanotechnology, Nanomaterials, Nanomedicine and Transfer 1619 May 2010, Bratislava, Slovakia.

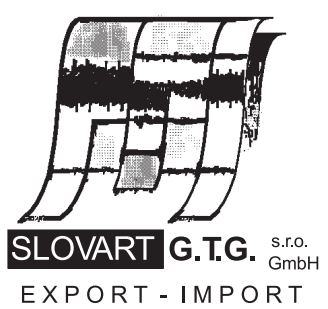

EXPORT - IMPORT
EXPORT - I M P OR T

of periodicals and of non-periodically printed matters, books and CD-ROMs

Krupinská 4 PO BOX 152, 85299 Bratislava 5, Slovakia tel: ++421 263839 472-3, fax: ++421 263839485 info@slovart-gtg.sk; http://www.slovart-gtg.sk

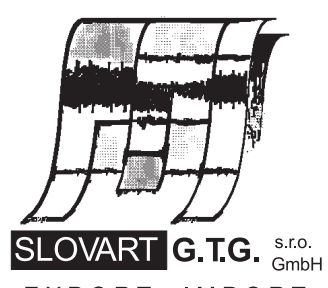

EXPORT - IMPORT 